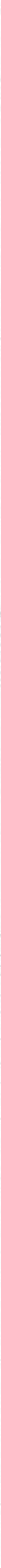





\title{
Negocios y sostenibilidad en la Base de la Pirámide ${ }^{1}$ \\ Business and Sustainability \\ at the Bottom of the Pyramid
}

Recibido: 17 de marzo de 2014

Evaluado: 28 de abril de 2014

Aceptado: 20 de junio de 2014

\author{
María Alejandra Pineda Escobar (Colombia) \\ Institución Universitaria Politécnico Grancolombiano \\ mapineda@poligran.edu.co \\ Maestría en Ciencias (MSc.) en Política Pública y Desarrollo Humano (Países Bajos) \\ y Maestría en Sostenibilidad y RSC (España)
}

\section{Resumen}

El objetivo de este artículo de reflexión es explorar la relación existente entre la actividad empresarial y la sostenibilidad en contextos de Base de la Pirámide (BdP), con el propósito de esclarecer el papel que el sector empresarial puede tener para el alivio de la pobreza. Comenzando con una revisión exhaustiva de la evolución de la teoría de la BdP, el artículo examina de manera crítica la Responsabilidad Social Empresarial (RSE) en contextos de pobreza, y sugiere la adopción de una interpretación más comprehensiva de la RSE que incorpore por completo la sostenibilidad (ambiental, económica y socialmente) en la operación del negocio. A continuación, se explora la

\begin{abstract}
This article reflects on the relationship there is between business activities and sustainability within Bottom of the Pyramid (BoP) contexts in order to clarify the role the corporate sector may have to decrease poverty. The article starts with an exhaustive revision of the BoP theory and critically analyzes the corporate social responsibility (CSR) with respect to poverty. It also suggests adopting a new comprehensive interpretation of the CSR that fully incorporates environmental, economical, and social sustainability within the business operation. Then, we explore the concept "sustainable business" (Hart, 2010) as an improved type of corporation that adopts the
\end{abstract}

1 La versión original de este artículo fue publicada inicialmente por Emerald Group Publishing como: Pineda-Escobar (2013) "Business, Sustainability and Base of the Pyramid. En Leonard and Gonzalez-Perez (Ed.) Principles and Strategies to Balance Ethical, Social and Environmental Concerns with Corporate Requirements. Advances in Sustainability and Environmental Justice, Volume 12, 67-91. ISSN: 2051-5030/doi:10.1108/S2051-5030(2013)0000012009 
noción de "empresa sostenible" (Hart, 2010) como un tipo mejorado de corporación que adopta el desarrollo sostenible, y se corresponde con los modelos de negocios para la BdP conocidos como negocios inclusivos. La sección final del artículo enfatiza la relevancia de los negocios inclusivos como empresas sostenibles con el potencial para mejorar de manera significativa las condiciones de vida para la mayoría de las comunidades pobres, siendo a su vez económicamente rentables y ambientalmente viables. Desde el punto de vista de la sostenibilidad, se sugiere que el mayor reto es alcanzar un balance entre un mayor consumo y producción en la $\mathrm{BdP}$, y la limitada capacidad ambiental de la Tierra.

Palabras clave: Base de la Pirámide, negocios inclusivos, empresa sostenible, América Latina, Responsabilidad Social Empresarial. sustainable development and corresponds to business models for BoPs known as inclusive businesses. The final part of the article makes emphasis on the importance of inclusive businesses as sustainable companies with the potential to improve life conditions significantly for the majority of unfortunate communities, becoming economically profitable and environmentally viable. Regarding sustainability, we suggest that the biggest challenge is to reach some balance between more consumption and BoP production and the environmental capacity of the Earth.

Keywords: Organizational values, social responsibility, public universities. 


\section{Introducción}

Mucho se ha escrito, analizado y debatido en torno a la idea de que el sector empresarial sea partícipe en el alivio de la pobreza. Argumentos a favor y en contra han surgido tanto desde la academia, como de la sociedad civil y de las empresas. Y a pesar de que se evidencia una mayor conciencia acerca de la urgencia de proveer una respuesta sólida a las necesidades de los billones de pobres que habitan en el mundo, no ha sido posible alcanzar un acuerdo acerca del papel de las compañías con respecto a la pobreza. Profundizar en ese debate no es el propósito de este artículo. Lo que se sabe con certeza es que es parte de los intereses, tanto de las compañías como de las comunidades, el lograr que las empresas se involucren con las sociedades de las que hacen parte. Porque, como fue expresado de una manera muy acertada por Björn Stigson, antiguo presidente del Consejo Empresarial Mundial para el Desarrollo Sostenible (WBCSD por sus siglas en inglés), "Los negocios no pueden tener éxito en sociedades que fracasan" (Stigson \& Rendlen, 2006).

En ese contexto, el objetivo del presente artículo de reflexión es explorar la relación existente entre la actividad empresarial y la sostenibilidad en contextos de Base de la Pirámide (BdP), con el propósito de esclarecer el papel que el sector empresarial puede tener para el alivio de la pobreza. Comenzando con una revisión detallada de la literatura disponible acerca de la Base de la Pirámide (BdP), la segunda sección del artículo presenta una cuidadosa revisión de los diez años de trabajo académico en el campo, ilustrando su evolución conceptual desde un concepto lineal a uno multifacético. A continuación, la tercera sección explora el concepto de desarrollo sostenible y expone la definición que es adoptada en este documento. La siguiente sección brinda un sucinto examen crítico de la evolución de la responsabilidad social empresarial (RSE) en lo que respecta a la $\mathrm{BdP}$, a través de la exploración de cómo la población pobre ha sido generalmente considerada por las compañías con fines altruistas, y cómo muy raramente las corporaciones han considerado la $\mathrm{BdP}$ como una posibilidad para la expansión del negocio o como una fuente de innovación y crecimiento. A continuación, se hace énfasis en la necesidad de ir más allá de la filantropía, resaltando la importancia de tener una comprensión más holística de la noción de RSE, que vaya más allá de un carácter de bienestar social incorrectamente inferido, y que incorpore por completo la sostenibilidad (ambiental, económica y social) en la operación del negocio.

El artículo continúa con un análisis del recientemente propuesto concepto de Negocios Inclusivos (NI), explorando sus implicaciones como un ejemplo de modelos sostenibles de negocios con el potencial de mejorar significativamente las condiciones de vida para la mayoría de las comunidades pobres, a la vez que son rentables económicamente y viables ambientalmente. Finalmente, la conclusión trae a colación algunas preguntas pendientes que resultan significativas y propone rutas para la profundización investigativa con miras al fortalecimiento de los negocios inclusivos con la BdP.

Evolución de la BdP de un concepto lineal a uno multifacético T1

C. K. Prahalad y Stuart Hart son los mayores proponentes del trabajo teórico sobre la Base de la Pirámide (BdP), comenzando con su trabajo seminal publicado en (2002), el cual acuñó la expresión Base de la Pirámide y allanó el camino para los 
últimos 10 años de trabajo académico en la materia. El concepto tiene su origen en la idea de un mercado insatisfecho e inexplorado en la base de la pirámide económica; haciendo énfasis en un fuerte interés comercial atado a la interpretación de la población con bajos ingresos solamente en términos de su potencial como posibles consumidores.

Desde allí, el concepto evolucionó hacia una interpretación más amplia que no se enfoca solamente en los pobres como potenciales consumidores, sino que también considera su participación significativa en las iniciativas de negocio a través de su integración efectiva a lo largo de la cadena de valor de las empresas, actuando como proveedores, distribuidores o socios de negocio.

\section{Distríbución geográfica y demográfica de la BdP}

En términos demográficos, la $\mathrm{BdP}$ representa un grupo poblacional de bajos ingresos, que vive en pobreza relativa y al cual no le es posible satisfacer algunas o incluso la mayoría de sus necesidades básicas. De acuerdo con Hammond A. et al. (2007), la metodología desarrollada en conjunto por el Banco Mundial, el Banco Interamericano de Desarrollo (BID) y el Instituto Mundial de Recursos (WRI por sus siglas en inglés) define la base de la pirámide como el grupo demográfico que percibe ingresos menores a $\$ 8$ al día, lo cual es equivalente a USD $\$ 3,000$ por persona por año en poder adquisitivo local. Según esta definición, la $\mathrm{BdP}$ representa un grupo de población de cuatro mil millones de

Figura 1. Distribución mundial de la BdP

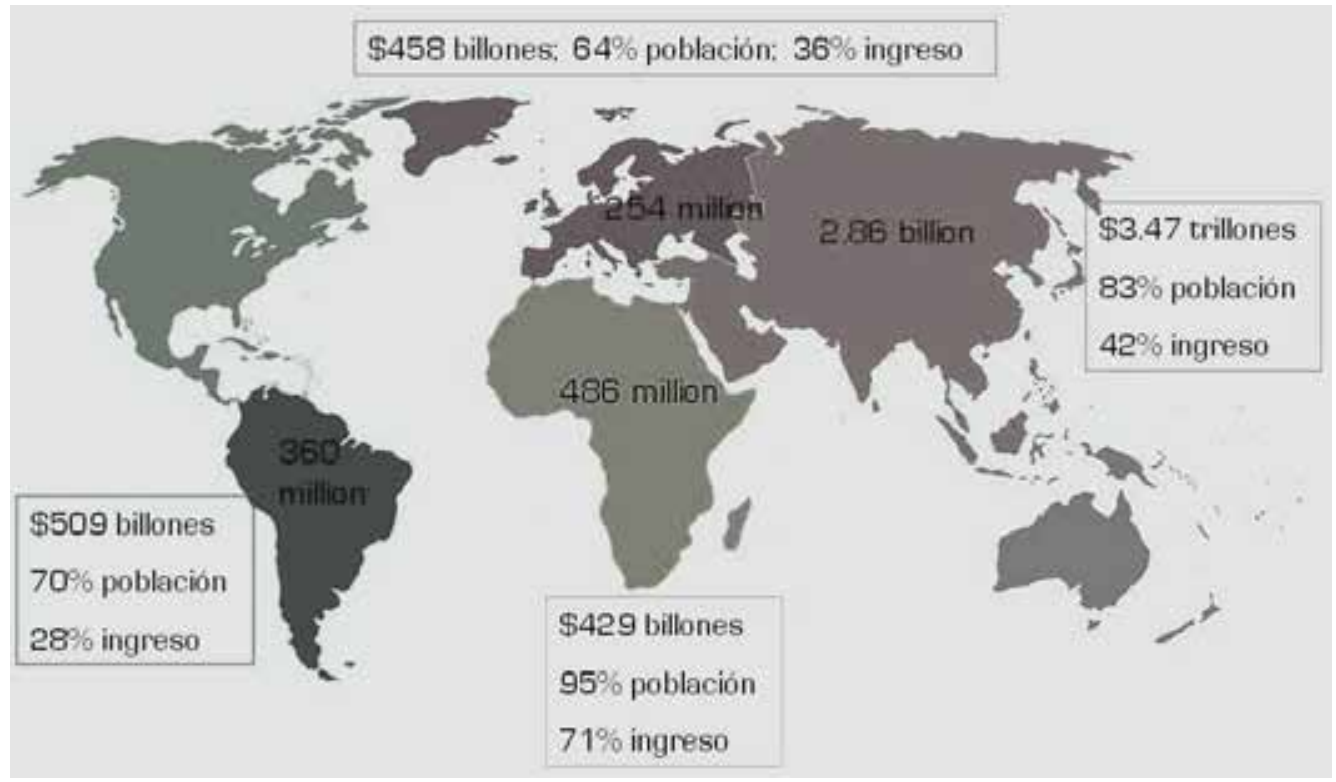

Fuente: Construcción propia basada en datos de Hammond, A. et al (2007). 
personas (cerca del 60\% de la población mundial actual, que alcanzó en 2012 el total de siete mil millones de personas) con un poder adquisitivo conjunto de USD $\$ 5$ trillones.

Como se muestra en la Figura 1, el mercado de la BdP se encuentra localizado principalmente en Asia, incluyendo el Medio Oriente, tanto en términos de población como de poder adquisitivo. América Latina corresponde al segundo mercado en términos de ingreso, con una cifra cercana a los $\$ 510$ mil millones de dólares y es, después de África, el tercero mayor en tamaño, con 360 millones de personas. A este respecto es interesante resaltar que América Latina es la región con la mayor brecha población-ingresos, dado que, a pesar de que esos 360 millones de personas representan el $70 \%$ de su población total, ganan de manera conjunta sólo el $28 \%$ del ingreso regional total, lo cual corrobora la enorme disparidad de distribución del ingreso que existe en los países de la región.

Ahora bien, habiendo aclarado la dimensión del grupo poblacional que constituye la base de la pirámide, es importante hacer énfasis en el carácter multidimensional de la pobreza, dado que no es solamente una falta de ingresos lo que estos cuatro mil millones de personas tienen en común. La población de la BdP vive en pobreza relativa y sin la posibilidad de responder a sus necesidades más básicas, lo que significa que carecen de acceso a "bienes, servicios y oportunidades económicas" fundamentales (Jenkins, B., E., Ishikawa, A., Geaneotes \& Paul, J., 2010, pág. 2).

El retrato de la BdP desarrollado por Hammond et al. identifica, además de sus bajos ingresos, varias características compartidas por la población de la BdP incluyendo múltiples necesidades básicas insatisfechas, puesto que "muchos viven en asentamientos informales (...) carecen de acceso al agua y servicios de saneamiento, electricidad y asistencia médica básica" (2007, pág. 18). A esto se suma su imposibilidad para garantizar acceso a la educación, su frecuente falta de nutrición adecuada y su dependencia de medios de vida de subsistencia y de pequeña escala.

Al comparar esta situación con los principios contenidos en la Declaración Universal de los Derechos Humanos,

Art. 3: Todo individuo tiene derecho a la vida, a la libertad y a la seguridad de su persona. (...) Art. 22: Toda persona, como miembro de la sociedad, tiene derecho a la seguridad social (...). Art. 25: 1.Toda persona tiene derecho a un nivel de vida adecuado que le asegure, así como a su familia, la salud y el bienestar y, en especial, la alimentación, el vestido, la vivienda, la asistencia médica y los servicios sociales necesarios (...). Art. 26: 1 . Toda persona tiene derecho a la educación. La educación debe ser gratuita, al menos en lo concerniente a la instrucción elemental y fundamental.

Hahn (2009, pág. 316) señala de manera precisa como las personas en la $\mathrm{BdP}$ claramente carecen de la mayoría de las necesidades básicas que deberían ser satisfechas de acuerdo con los derechos humanos de la DUDH.

Sin embargo, relacionar el tema de los derechos humanos con los negocios es una tarea compleja y algunas precauciones importantes deben ser tenidas en cuenta. Como es ilustrado de manera ejemplar por Whelan, Moon y Orlitzky (2009, pág. 372), la más palpable es el hecho de que el carácter absoluto y universal de los 
derechos humanos no deja de tener cuestionamientos. De hecho, como es señalado por los autores, "deberíamos (...) ser cautelosos al presumir que existe algún tipo de consenso a nivel mundial respecto a todos y cada uno de los derechos listados en la Declaración Universal de los Derechos Humanos (DUDH)". Adicionalmente, los derechos humanos son criticados por su carácter relativamente impreciso, mientras que algunos aseguran que la DUDH es un ideal enfocado en el Occidente.

Hecha esta salvedad, es apropiado afirmar que una creciente conciencia pública respecto al comportamiento corporativo es evidente, manifestada por ejemplo en la creciente preocupación alrededor de las violaciones de los derechos humanos por parte de las corporaciones. Anteriormente, los derechos humanos se consideraban bajo la responsabilidad exclusiva de los gobiernos y las organizaciones internacionales que los agrupan; pero las últimas décadas han traído consigo insistentes llamados al sector privado para que se involucre de manera activa y se haga responsable de materias sociales y de desarrollo. En ese sentido, se percibe al sector empresarial teniendo una participación relevante para el logro de los Objetivos de Desarrollo del Milenio (ODM) e iniciativas tangibles han madurado en el seno de las Naciones Unidas (ONU), específicamente el Pacto Mundial y el nombramiento del 'Representante Especial del Secretario General en el tema de derechos humanos y corporaciones transnacionales y otras iniciativas de negocios'.

Esta visión debe ser leída como un rol complementario del Estado y el sector privado en respuesta a déficits sociales, y no debería ser malinterpretada como un reemplazo total del primero por parte del último. Lo anterior es referido por Maak
(2009) como una "corresponsabilidad política activa” (pág. 370) de los ciudadanos corporativos, en particular para aquellas situaciones relacionadas con justicia social y sostenibilidad ambiental que hasta ahora no han recibido una respuesta adecuada por parte de los gobiernos. Sin embargo, esta corresponsabilidad activa debe ser contrastada con la noción de que las compañías tienden a enfocarse en las utilidades. Por ende, cuando se inste a las corporaciones a lidiar con déficits sociales, es necesario reconocer el hecho de que sus actividades como instituciones de negocios están enmarcadas dentro de su objetivo de creación de valor económico. Por lo tanto, el compromiso activo de una corporación con la BdP debe ser el resultado de un interés propio del negocio que esté inmerso en su compromiso y su corresponsabilidad con la sociedad de la cual hace parte.

De esta manera, reconociendo la pobreza como un fenómeno multidimensional determinado no solamente por factores económicos sino también por fuerzas políticas y sociales que inhiben el desarrollo humano y el potencial empresarial (Tashman \& Marano, 2010), los estudiosos de la BdP proponen una aproximación diferente al problema de la pobreza en estas sociedades, acercándose al mismo desde una nueva perspectiva de mercado. El argumento es que ambientes como estos, caracterizados por la falla de los derechos humanos dentro de sociedades política y económicamente débiles, pueden proveer al sector empresarial con nuevas oportunidades de mercado, con el potencial para mejorar estos problemas sociales al mismo tiempo que son económicamente rentables para la compañía. Un argumento central es que los pobres no deben seguir siendo vistos como entes pasivos caracterizados por sus carencias, sino que deberían ser 
entendidos como miembros activos de la sociedad que son también capaces de comprometerse con sus propios procesos para salir de la pobreza.
La tabla No. 1 resume las principales contribuciones que han caracterizado la evolución del trabajo teórico en el campo de la BdP.

Tabla No. 1. Evolución del trabajo teórico en la BdP

\begin{tabular}{|c|c|c|}
\hline Año & Autor(es) & Contribución principal \\
\hline 2002 & $\begin{array}{l}\text { Prahalad, C.K \& } \\
\text { Hart, S. }\end{array}$ & $\begin{array}{l}\text { Artículo "The Fortune at the Bottom of the Pyramid" ("La fortuna en la base de } \\
\text { la pirámide"). Considerado el trabajo seminal acerca de la BdP, corresponde a la } \\
\text { primera publicación acerca del tema que provee la base fundamental para el } \\
\text { desarrollo conceptual de la BdP.. }\end{array}$ \\
\hline 2002 & $\begin{array}{l}\text { Prahalad, C.K \& } \\
\text { Hammond, A. }\end{array}$ & $\begin{array}{l}\text { Artículo "Serving the world's poor, profitably" ("Sirviendo a los pobres del mundo, } \\
\text { rentablemente"). En línea con la publicación previa, los autores hacen énfasis en } \\
\text { lógica empresarial existente tras la participación del sector privado en comunidades } \\
\text { pobres de la base de la pirámide. }\end{array}$ \\
\hline 2002 & $\begin{array}{l}\text { Hart, S. \& } \\
\text { Christensen, C. }\end{array}$ & $\begin{array}{l}\text { Enfocando el análisis en el tema de la innovación, Hart y Christensen presentan el } \\
\text { artículo "The Great Leap, driving innovation from the base of the pyramid" ("El gran } \\
\text { salto, impulsando la innovación desde la base de la pirámide"), el cual amplía el } \\
\text { concepto de innovación disruptiva acuñado por Christensen (1997) y lo propone } \\
\text { como una característica esencial de los esfuerzos de negocios que apuntan a la } \\
\text { base de la pirámide. }\end{array}$ \\
\hline 2005 & Prahalad, C.K & $\begin{array}{l}\text { Prahalad publica el libro titulado "The Fortune at the Bottom of the Pyramid: } \\
\text { Eradicating Poverty through Profits" (“La fortuna en la base de la pirámide: } \\
\text { erradicando la pobreza a través de la rentabilidad”). En este trabajo, el autor detalla } \\
\text { las virtudes de los modelos de negocio orientados a la población más vulnerable, } \\
\text { presentando una serie de casos de estudio exitosos en varios países en vía de } \\
\text { desarrollo. }\end{array}$ \\
\hline 2005 & $\begin{array}{l}\text { Hart, S.L. \& London, } \\
\text { T. }\end{array}$ & $\begin{array}{l}\text { En su artículo "Developing Native Capability, what multinational corporations can } \\
\text { learn from the base of the pyramid" ("Desarrollando capacidades nativas, lo que } \\
\text { pueden aprender las corporaciones multinacionales de la base de la pirámide"), Hart } \\
\text { y London desarrollan el concepto de 'capacidades nativas' e invitan a las compañías } \\
\text { a evitar la imposición de estrategias extranjeras, entendiendo el contexto local y } \\
\text { construyendo confianza y capital social con la población de la BdP. }\end{array}$ \\
\hline 2007 & $\begin{array}{l}\text { Anderson, J. \& } \\
\text { Billou, N. } \\
\text { Anderson, J. \& } \\
\text { Markides, C. }\end{array}$ & $\begin{array}{l}\text { Artículos "Serving the World's Poor: Innovation at the Base of the Economic } \\
\text { Pyramid" ("Sirviendo a los pobres del mundo: innovación en la base de la pirámide") } \\
\text { y "Strategic Innovation at the base of the pyramid" ("Innovación estratégica en la } \\
\text { base de la pirámide"). Los autores presentan el marco conceptual de las } 4 \text { As [por sus } \\
\text { iniciales en inglés: disponibilidad, asequibilidad, conciencia y aceptabilidad], como } \\
\text { un marco de trabajo para la innovación estratégica, detallando las áreas clave en las } \\
\text { que las compañías actuando en la BdP deben enfocarse para ser exitosas. }\end{array}$ \\
\hline 2007 & Hammond, A. et al. & $\begin{array}{l}\text { Publicación conjunta del World Resources Institute y la Corporación Financiera } \\
\text { Internacional titulada "The next } 4 \text { billion, market size and business strategy at the } \\
\text { base of the pyramid" ("Los próximos cuatro mil millones, tamaño del mercado y } \\
\text { estrategia de negocios en la base de la pirámide"). Presenta estimados empíricos } \\
\text { acerca del poder adquisitivo de la población de la BdP como un todo, y acerca de } \\
\text { sus comportamientos como consumidores. Así mismo, brinda una mirada al estado } \\
\text { de los mercados de la BdP en el mundo e identifica cuatro estrategias principales } \\
\text { que caracterizan a las compañías que están teniendo éxito en dichos mercados. }\end{array}$ \\
\hline
\end{tabular}




\begin{tabular}{|c|c|c|}
\hline Año & Autor(es) & Contribución principal \\
\hline 2007 & Karnani, A. & $\begin{array}{l}\text { En su artículo "Misfortune at the Bottom of the Pyramid" ("Infortunio en la base } \\
\text { de la pirámide"), el autor provee una de las críticas más citadas a la teoría acerca } \\
\text { del potencial comercial de la BdP. Karnani llama la atención sobre la necesidad de } \\
\text { aumentar realmente los ingresos de los pobres en lugar de simplemente enfocarse } \\
\text { en su potencial como consumidores. }\end{array}$ \\
\hline 2008 & $\begin{array}{l}\text { Mendoza, R. \& } \\
\text { Thelen, N. }\end{array}$ & $\begin{array}{l}\text { El artículo "Innovations to Make Markets More Inclusive for the Poor" ("Innovaciones } \\
\text { para hacer los mercados más incluyentes para los pobres") propone una tipología } \\
\text { de las estrategias e innovaciones que han contribuido a hacer los mercados más } \\
\text { incluyentes para los pobres, no sólo en lo que respecta a su acceso a dichos } \\
\text { mercados, sino también en lo relacionado con su participación significativa en } \\
\text { formas que incrementen su empoderamiento y desarrollo humano. }\end{array}$ \\
\hline 2008 & Simanis, E. \& Hart, S. & $\begin{array}{l}\text { A través de la publicación “The Base of the Pyramid Protocol: Toward Next } \\
\text { Generation BoP Strategy" (“El protocolo Base de la Pirámide: Hacia una estrategia } \\
\text { BdP de próxima generación”), los autores presentan una crítica a lo que ellos llaman } \\
\text { estrategias para la BdP 'de primera generación' (BdP 1.0), que han estado enfocadas } \\
\text { en explotar comercialmente los mercados de la base de la pirámide. Y en respuesta, } \\
\text { proponen las estrategias 'de segunda generación' (BdP 2.0), basadas en un diálogo } \\
\text { con gente local para permitir la invención y creación conjuntas de oportunidades } \\
\text { de negocio. }\end{array}$ \\
\hline 2008 & $\begin{array}{l}\text { Kandachar, P. \& } \\
\text { Halme, M. (Ed.) }\end{array}$ & $\begin{array}{l}\text { "Sustainability Challenges and Solutions at the Base of the Pyramid: Business, } \\
\text { Technology and the Poor" ("Retos y soluciones de sostenibilidad en la base de la } \\
\text { pirámide: negocios, tecnología y los pobres") ofrece una recopilación de ensayos } \\
\text { escritos por autores altamente renombrados de países desarrollados y en vía } \\
\text { de desarrollo, quienes proveen un nutrido análisis de los potenciales retos y } \\
\text { oportunidades creadas por la BdP, y exploran rutas de acción futura que pueden } \\
\text { llevar a un desarrollo sostenible para los pobres. }\end{array}$ \\
\hline 2009 & Hahn, R. & $\begin{array}{l}\text { El artículo "The Ethical Rational of Business for the Poor - Integrating the concepts } \\
\text { Bottom of the Pyramid, Sustainable Development, and Corporate Citizenship" ("Las } \\
\text { justificativos éticos de los negocios para los pobres - integrando los conceptos Base } \\
\text { de la Pirámide, desarrollo sostenible y ciudadanía corporativa"), conecta la idea } \\
\text { de negocios en la base de la pirámide con el concepto de desarrollo sostenible, } \\
\text { particularmente con la noción de justicia inter e intrageneracional. }\end{array}$ \\
\hline 2009 & Simanis, E. \& Hart, S. & $\begin{array}{l}\text { Artículo "Innovation from the inside out" ("Innovación desde el interior"). } \\
\text { Apoyándose en la experiencia ganada en la investigación acerca del "Protocolo de } \\
\text { la BdP", los autores proponen el 'paradigma de innovación arraigada' como la mejor } \\
\text { manera en la cual las compañías pueden generar confianza y ganar intimidad en sus } \\
\text { negocios, generando una ventaja competitiva sostenible que resulta apropiada para } \\
\text { los mercados de la BdP. }\end{array}$ \\
\hline 2010 & Hart, S. & $\begin{array}{l}\text { En la tercera edición de "Capitalism at the crossroads, next generation business } \\
\text { strategies for a post-crisis world" ("Capitalismo en la encrucijada, estrategias de } \\
\text { próxima generación para un mundo post-crisis"), Hart se enfoca en el mundo } \\
\text { después de la crisis financiera y propone un modelo de capitalismo sostenible, } \\
\text { basado en un nuevo paradigma de negocios que es socialmente responsable y } \\
\text { viable ambientalmente, enfocándose en la población de la BdP. }\end{array}$ \\
\hline 2011 & $\begin{array}{l}\text { Berger, R. Choi, C. \& } \\
\text { Kim, J. }\end{array}$ & $\begin{array}{l}\text { En el artículo "Responsible Leadership for Multinational Enterprises in Bottom of } \\
\text { Pyramid Countries: The Knowledge of Local Managers" ("Liderazgo responsable } \\
\text { para empresas multinacionales en países de la base de la pirámide: el conocimiento } \\
\text { de los administradores locales"), los autores analizan y hacen énfasis en valorar el } \\
\text { conocimiento, identidad y empuje de los administradores locales, en lugar de tener } \\
\text { una dependencia excesiva en liderazgo expatriado cuando se tienen operaciones } \\
\text { de negocios en mercados de la base de la pirámide. }\end{array}$ \\
\hline
\end{tabular}




\begin{tabular}{|c|l|l|}
\hline Año & \multicolumn{1}{|c|}{ Autor(es) } & \multicolumn{1}{c|}{ Contribución principal } \\
\hline 2012 & $\begin{array}{l}\text { Halme, M. } \\
\text { Lindeman, S \& } \\
\text { Linna, P. }\end{array}$ & $\begin{array}{l}\text { En su artículo "Innovation for Inclusive Business: Intrapreneurial } \\
\text { Bricolage in Multinational Corporations" ("Innovación para negocios inclusivos: } \\
\text { bricolaje intraemprendedor en las corporaciones multinacionales"), los autores } \\
\text { introducen el concepto de 'bricolaje intraemprendedor' para analizar los aspectos } \\
\text { internos de la gestión organizacional que pueden impulsar o frenar la innovación en } \\
\text { modelos de negocios en la BdP. }\end{array}$ \\
\hline 2012 & $\begin{array}{l}\text { Schuster, T. \& } \\
\text { Holtbrügge, D. }\end{array}$ & $\begin{array}{l}\text { Recurriendo a una aproximación metodológica de múltiples casos de estudio, los } \\
\text { autores del artículo titulado "Market entry of multinational companies in markets } \\
\text { at the bottom of the pyramid: A learning perspective" ("Entrada al mercado de } \\
\text { compañías multinacionales en mercados en la base de la pirámide: una perspectiva } \\
\text { de aprendizaje") analizan las actividades de corporaciones multinacionales (EMNs) } \\
\text { en mercados de la BdP, desde una perspectiva de internacionalización. }\end{array}$ \\
\hline 2012 & $\begin{array}{l}\text { El artículo "Inclusive business, human rights and the dignity of the poor: a glance } \\
\text { beyond economic impacts of adapted business models" ("Negocios inclusivos, } \\
\text { derechos humanos y la dignidad de los pobres: una mirada más allá de los impactos } \\
\text { económicos de los modelos de negocio adaptados"), explora las conexiones entre } \\
\text { la dignidad humana y los modelos de negocios inclusivos, más allá de los resultados } \\
\text { puramente económicos. }\end{array}$ \\
\hline Hahn, R. &
\end{tabular}

Fuente: construcción propia

Para resumir, podemos identificar de manera general dos estadios principales en la evolución del concepto de la BdP: i.) el primer estadio surge de las aproximaciones iniciales hechas por los profesores Hart y Prahalad, cuando la BdP fue concebida como un concepto unidimensional. Su carácter central mostró un interés fuertemente comercial, entendiendo la población de bajos ingresos solamente en términos de mercado, como un segmento consumidor no explotado que representa una "fortuna" potencial. Por tanto, la BdP fue primordialmente presentada como una nueva oportunidad de negocios para el sector privado. ii.) El segundo estadio evolucionó hacia una interpretación multidimensional de la BdP, ampliando el espectro de análisis y proveyendo más y más variadas posibilidades de acción para la población de bajos ingresos. De esta manera, la $\mathrm{BdP}$ pasó de ser entendida únicamente desde la perspectiva de la demanda, hacia una interpretación holística que incluye opciones que describen a los pobres como potenciales empleados, distribuidores, proveedores o socios de negocios. Este entendimiento más amplio de la BdP corresponde a los modelos de negocios inclusivos, que serán discutidos en la tercera sección del presente artículo.

\section{Sobre el desarrollo sostenible}

Al igual que la responsabilidad social empresarial, el concepto de desarrollo sostenible continúa siendo debatido, refutado y reinterpretado en la literatura nacional e internacional. No siendo propósito de este documento el profundizar en un análisis conceptual de la materia, no se ahondará en dicha discusión. Sin embargo, si es necesario esclarecer las razones que han llevado a la sociedad humana a interesarse por estas temáticas; y exponer lo que en este artículo se entiende por desarrollo sostenible.

La crisis ambiental que enfrenta la humanidad ha sido ampliamente analizada y documentada tanto en la academia como en la literatura convencional (p. ej. Brown, 2011; Goodland, 1994; Ludevid, 
1997; Velásquez, 2008). Para citar algunos ejemplos podemos recurrir a argumentos como los de Hawken, Lovins \& Lovins (1999), quienes estiman que durante los últimos treinta ańos del siglo $\mathrm{XX}$, cerca de un tercio de todos los recursos naturales del planeta fueron consumidos, llevando muchos de éstos a su extinción. Por su parte, Leonard (2010) y otros han calculado que la huella ecológica de la humanidad excede la capacidad de carga de la Tierra, siendo esta cifra para inicios del siglo XXI cercana a $25 \%$. Tal desbalance lo reflejan Wackernagel y Rees, al estimar que si en todos los países tuviésemos los mismos niveles de consumo que manejan las naciones industrializadas, se haría necesario contar con el equivalente a cuatro planetas Tierra para suplir tan amplio nivel de demanda de recursos (Boada, Rocchi y Kuhndt, 2005). De la misma manera, las consecuencias del cambio climático se reflejan en afectaciones en diversos ecosistemas mundiales, incluyendo degradación de suelos, desertificación, deshielo, y alteraciones en los ciclos de producción agrícola; con implicaciones no sólo en la sostenibilidad ambiental, sino también en la geopolítica internacional y en las capacidades de asimilación de dichos cambios por las sociedades humanas (Brown, 2011; Dalby, 2013).

En respuesta a este desbalance, en las últimas décadas del siglo XX surge una tendencia de análisis del desarrollo que busca armonizar el crecimiento económico, con las necesidades sociales y la eficiencia ambiental; que ha pasado a ser reconocida como el desarrollo sostenible. Para los propósitos de este artículo de reflexión, adoptaremos la definición de desarrollo sostenible de mayor aceptación en esferas académicas y políticas, la cual corresponde a aquella dada por Gro Harlem Bruntland en el año de 1987, al liderar para la Organización de las Naciones
Unidas (ONU), la Comisión Mundial de Medio Ambiente y Desarrollo (WCED por sus siglas en inglés), conocida en la historia de la sostenibilidad como la Comisión Brundtland. El reporte denominado "Nuestro Futuro Común" (ONU, 1987), fue publicado por esta Comisión brindando por primera vez en la agenda internacional una definición al término desarrollo sostenible, así: "Desarrollo que busca satisfacer las necesidades de las generaciones presentes sin comprometer las posibilidades de las futuras generaciones para atender sus propias necesidades".

Complementando esta definición, y abordándola desde una perspectiva de justicia inter e intrageneracional, es preciso reconocer junto con Montes (2013), que el desarrollo sostenible debe propender por una correcta administración de los recursos, que tome en consideración las limitaciones de los bienes y servicios, no sólo en términos ambientales sino también económicos y socio-culturales. Tal reconocimiento de la importancia de los aspectos socio-culturales para abordar el desarrollo sostenible, resulta fundamental para comprender la sostenibilidad empresarial en la BdP, toda vez que como se expuso en el apartado anterior, los mercados de la $\mathrm{BdP}$ se caracterizan por unas condiciones socio-económicas y culturales específicas y muchas veces endógenas a tales contextos.

\section{Acerca de la Responsabilidad Social Empresarial en la Base de la Pirámide}

A pesar de que hay una aceptación creciente, tanto en países desarrollados como en vía 
de desarrollo, del hecho que las compañías tienen responsabilidades que van más allá de sus accionistas para con un espectro más amplio de grupos de interés, la idea de la responsabilidad social empresarial (RSE) sigue siendo un concepto controversial y polisémico, encontrando sus críticos más reconocidos en los trabajos de Friedman, Henderson y Reich (Friedman, 1970; Henderson, 2001; Reich 1998).

Desde la perspectiva de los proponentes de la RSE, las décadas recientes ofrecen un nutrido compendio de literatura académica y gerencial con múltiples aproximaciones y reinterpretaciones conceptuales, cuya exploración detallada está más allá del propósito del presente artículo de reflexión (ver por ejemplo Dahlsrud, 2006, Freeman \& Hasnaoui, 2011; Garriga \& Mele, 2004; Gond \& Moon, 2011; Visser, Matten, Pohl, $\&$ Tolhurst, 2010). Sin embargo, es posible percibir un terreno común entre todas las variadas definiciones que va en línea con lo propuesto por Carroll (uno de los estudiosos más reconocidos en este campo) en 1991, entendiendo las responsabilidades de los negocios como aquellas que emanan de cuatro dominios interrelacionados, a saber: el económico, el legal, el ético y el discrecional (filantrópico), y reconociendo la existencia de múltiples grupos de interés.

La Organización Internacional de Normalización (ISO) en su reciente Guía acerca de la responsabilidad social (2010), la define como:

La responsabilidad de una organización sobre los impactos de sus decisiones y actividades en la sociedad y el ambiente, a través de un comportamiento transparente y ético que:

- contribuye al desarrollo sostenible, incluyendo la salud y el bienestar de la sociedad;
- toma en cuenta las expectativas de los interesados;

- cumple con las leyes aplicables y es consistente con las normas internacionales de comportamiento y

- está integrada a lo largo de la organización y se practica en sus relaciones.

De otro lado, considerando las contribuciones por parte de estudiosos y administradores de países en vía de desarrollo, es importante resaltar que dado el carácter desigual y diverso de las naciones del hemisferio norte y sur, cualquier iniciativa de RSE debe ser sensible al contexto; tomando en cuenta posibles diferencias económicas, sociales, políticas, ambientales, culturales y organizacionales (Barkemeyer, 2011). De igual manera, al explorar el argumento generalmente aceptado de que la RSE se traduce en buenas prácticas de negocios que permiten que las compañías "hagan bien haciéndolo bien" (Prahalad, 2005), los estudiosos de la filosofía moral alertan acerca de la necesidad de reconocer la carencia de un acuerdo globalmente aceptado sobre qué constituye 'buenas prácticas' en primer lugar; lo cual resalta la necesidad de que la RSE sea sensible a la existencia de múltiples visiones respecto a la moralidad (Eabrasu, 2012).

Dado todo lo anterior, tal como acertadamente lo exponen Gond y Moon, la RSE es inherentemente "dinámica, superpuesta y contextual” (2011, pág. 3), así que se la interpreta mejor como un constructo sombrilla (un concepto comprehensivo que explica un conjunto diverso de fenómenos (ver Hirsh \& Levin, 1999)) cubriendo un amplio rango de fenómenos en las relaciones entre los negocios y la sociedad.

Habiendo ilustrado las complejidades de la RSE, es pertinente explorar también la relación entre filantropía y RSE. El rol 
jugado por el sector privado en el alivio de la pobreza, particularmente aquel de las corporaciones multinacionales, ha sido sujeto de gran debate a nivel internacional tanto en espacios académicos como no académicos (Frynas, 2005; Frynas, 2008; Jamali, 2010; Lodge \& Wilson, 2006; Muthuri, Moon, \& Idemudia, 2012; VanSandt \& Sud, 2012). En este escenario, como lo ilustra Barkemeyer (2011), la literatura académica sobre la relación entre el desarrollo y la RSE ha cuestionado la idoneidad de los "regímenes actuales de RSE para afrontar de manera efectiva algunos de los retos más apremiantes para la sostenibilidad global” (pág. 257).

Por regla general, lo que parece suceder en respuesta a las necesidades sociales características de países en desarrollo y menos desarrollados, es que las corporaciones y las organizaciones sin ánimo de lucro tienden a malinterpretar la RSE como equivalente a donaciones puramente filantrópicas por parte de las empresas para promover el bien social. En este sentido, es de la mayor importancia enfatizar que las compañías no deben entender la RSE como su forma de devolver a la sociedad, como si le hubiere substraído algo en primer lugar. Por el contrario, la RSE debe ser adoptada como la manera de actuar como parte de la sociedad, desde una perspectiva ganar-ganar que exhibe una lógica de mutuo beneficio. El pensar: ambos (i.e. la empresa y la sociedad) nos beneficiamos, es radicalmente opuesto a una línea de pensamiento que sugiere que una compañía se beneficia a expensas de la sociedad, de donde se desprende que es su deber devolver algo a la misma; como si se estuviese pagando por haber cometido un pecado.

Cuando las corporaciones caen en esta interpretación paternalista y equivocada, en la mayor parte de los casos terminan llevando a cabo actividades filantrópicas - pero desligadas de su quehacer empresarial- que tienen la desventaja de ser percibidas como una forma de remordimiento corporativo o como un intento de apaciguar la conciencia corporativa (Cooke, 2010). De manera similar, las iniciativas altruistas son consideradas en ocasiones únicamente como una estrategia de relaciones públicas que apunta a mejorar la reputación corporativa y a ganar una licencia para operar (Gates \& Steane, 2009). De hecho, algunas visiones críticas acerca de intervenciones de RSE por parte de corporaciones multinacionales en países en vía de desarrollo y menos desarrollados, ponen en duda su legitimidad y consideran que dichas intervenciones:

aún tienden a estar diseñadas para garantizar una rendición de cuentas frente a los consumidores en países desarrollados, organizaciones de denuncia y medios de comunicación, con el fin de mantener ante sus ojos la legitimidad de las corporaciones multinacionales (Jeppesen $\&$ Lund-Thomsen, 2010, pág.141).

Por otro lado, tanto el grado de efectividad de las donaciones filantrópicas, como su real contribución a la reducción de la pobreza, han sido sujeto de cuestionamientos (Canel, Idemudia, \& North, 2010; Ite, 2005; Singer, 2006). Tal como lo señalan Kolk y van Tulder (2006), la sostenibilidad de las respuestas del sector privado que van dirigidas a los pobres puede ser alcanzada solamente al "ofrecer a los pobres 'herramientas' (conocimiento técnico, tecnología, recursos) para escapar de la trampa de la pobreza por ellos mismos" (pág. 797). Estas herramientas, sin embargo, aún no están siendo ofrecidas por completo a los pobres por parte de las corporaciones. De 
esta manera, se da piso a percepciones de una falta de interés verdadero en el desarrollo de la comunidad en el largo plazo, $\mathrm{y}$ al uso abusivo del poder corporativo.

\section{La migración hacia una comprensión más holística de la RSE: yendo más allá de la filantropía en la BdP}

En respuesta a esta idea equivocada acerca de la RSE, varios autores han argumentado a favor de una evolución conceptual que nos lleve a una interpretación más holística de la noción de RSE. Tal evolución ha sido renombrada por algunos autores como responsabilidad y sostenibilidad empresarial (manteniendo el acrónimo RSE), o RSE 2.0 (Visser et al., 2010), haciendo énfasis en su inherente triple cuenta de resultados, y yendo más allá de un carácter puramente social, erróneamente inferido en la actualidad.

La RSE 2.0 implica, por tanto, dar consideración completa a la sostenibilidad cuando se hacen negocios, respondiendo a todas las cuestiones sociales, económicas y ambientales. De esta manera, la RSE viene a estar integrada a lo largo de la compañía, permeando cada práctica organizacional. Y las relaciones entre la empresa y la sociedad se mueven más allá de la donación filantrópica lineal, hacia relaciones más proactivas e interactivas que dejan de ver a las corporaciones como donantes paternalistas y a las comunidades como meros receptores pasivos. En las palabras de Visser, la "RSE 2.0 se trata de diseñar $\mathrm{y}$ adoptar un modelo de negocios inherentemente sostenible y responsable" (Visser et al., 2010, pág. 127).

Esta comprensión holística de la RSE se compagina con lo que Stuart Hart presenta como una 'empresa sostenible', aquella con la habilidad de generar ideas de negocio rentables, que pueden al mismo tiempo "mejorar la calidad de vida de los pobres del mundo, respetar la diversidad cultural, inspirar a los empleados, construir comunidades y conservar la integridad ecológica del planeta para futuras generaciones" (Hart, 2010, pág. 17). Así, una empresa sostenible es un tipo nuevo y mejorado de corporación que acoge el desarrollo sostenible. En lugar de ver sus responsabilidades sociales como medios para obtener un objetivo - licencia pública para operarentiende la sostenibilidad, y por tanto la RSE, como la razón de ser de la operación empresarial. Adopta la sostenibilidad como la única estrategia efectiva para la perdurabilidad corporativa. Por lo tanto, ve la obtención de utilidades como un resultado de su compromiso responsable con la sociedad, y no lo contrario.

Llevar el concepto de empresas sostenibles al terreno de la BdP implica un movimiento claro de las relaciones empresa-sociedad más allá de la filantropía. Un movimiento claro que, a su vez, resalta cómo la oportunidad para la participación activa de las compañías en la BdP implica el uso de nuevos modelos de negocios que permitan mejorar de manera significativa las condiciones humanas y ambientales para las regiones más pobres el mundo, a la vez que se genera rentabilidad para el negocio. Alcanzar esto requeriría reducir la focalización en el crecimiento del consumo, trasladando el énfasis hacia la elevación de los estándares de vida de los pobres, a través de una estrategia de negocios que permita la cobertura de las necesidades básicas insatisfechas de una manera que sea económicamente efectiva y ecológicamente viable; es decir, una estrategia de sostenibilidad. 
Un ejemplo de este tipo de empresas sostenibles para la $\mathrm{BdP}$ es el esquema conocido como Negocios Inclusivos (NI): modelos de negocio sostenibles con el potencial de mejorar significativamente las condiciones de vida de la mayoría de las comunidades pobres, al mismo tiempo que son económicamente rentables y ambientalmente viables.

\section{Los Negocios Inclusivos como empresas sostenibles en la Base de la Pirámide}

De acuerdo con la definición dada por la Alianza para los Negocios Inclusivos, un negocio inclusivo es una iniciativa de negocios que busca construir puentes entre el negocio y las poblaciones de bajos ingresos para el beneficio de ambos (SNV-WBCSD, 2008). En otras palabras, los NI son modelos de negocio sostenibles que apuntan a mercados en la BdP. A través de la inclusión efectiva de las comunidades más pobres dentro de sus cadenas de valor, el sector privado provee ocupaciones y riqueza a las comunidades de bajos ingresos, al mismo tiempo que genera utilidades para la compañía.

De esta manera, los NI son proyectados como una estrategia de negocio que más allá de encarnar el paternalismo o la filantropía, empodera a las poblaciones vulnerables, vinculando necesidades sociales a la creación de trabajos y fuentes estables de ingreso; ofreciendo un mecanismo más efectivo para reducir la pobreza y promover el desarrollo en el largo plazo.

El cuadro No. 1 presenta un compendio no exhaustivo de varias definiciones dadas al concepto de negocios inclusivos. Como se desprende de su lectura, las diferentes definiciones no alteran de manera significativa la interpretación del concepto y, con tonos similares, todas se enfocan principalmente en resaltar un acercamiento proactivo de mercado para superar la pobreza, basado en una administración sostenible de la cadena de valor.

Cuadro No. 1. Definiciones seleccionadas de negocios inclusivos.

\begin{tabular}{|c|l|}
\hline Proponente & \multicolumn{1}{c|}{ Definición } \\
\hline Alianza SNV- WBCSD & $\begin{array}{l}\text { Negocio que busca contribuir al alivio de la pobreza mediante la inclusión de las } \\
\text { comunidades de bajos ingresos en su cadena de valor, sin perder de vista el objetivo final } \\
\text { de la empresa, que es la generación de ganancias. (SNV-WBCSD, 2008) }\end{array}$ \\
\hline WBCSD & $\begin{array}{l}\text { Soluciones de negocio sostenibles que van más allá de la filantropía y amplían el acceso a } \\
\text { bienes, servicios y oportunidades de subsistencia para las comunidades de bajos ingresos } \\
\text { en formas comercialmente viables; tratando de encontrar sinergias entre los objetivos de } \\
\text { desarrollo y las operaciones de negocio de la compañía. (http://www.inclusive-business. } \\
\text { org/inclusive-business.html) }\end{array}$ \\
\hline PNUD & $\begin{array}{l}\text { Modelos de negocio que incluyen a los pobres en varios puntos de la cadena de valor: } \\
\text { en el lado de la demanda como clientes y consumidores, y en el lado de la oferta como } \\
\text { empleados, productores y propietarios de negocios. Construyen puentes entre las } \\
\text { empresas y las personas pobres para beneficio mutuo en la cadena de suministro, en el } \\
\text { lugar de trabajo y en el mercado. (PNUD, 2010) }\end{array}$ \\
\hline CFI & $\begin{array}{l}\text { Modelos de negocios que amplían el acceso a bienes, servicios y oportunidades de } \\
\text { subsistencia para aquellos en la base de la pirámide económica mundial (BdP), en formas } \\
\text { comercialmente viables y escalables. (IFC, 2010) }\end{array}$ \\
\hline
\end{tabular}




\begin{tabular}{|c|l|}
\hline Proponente & \multicolumn{1}{c|}{ Definición } \\
\hline Fundación AVINA & $\begin{array}{l}\text { Iniciativas rentables y ambiental y socialmente responsables, que buscan mejorar, a través } \\
\text { de las fuerzas del mercado, la calidad de vida de las comunidades de bajos ingresos, } \\
\text { fomentando su participación en todas las fases de la cadena de valor. (http://www.avina. } \\
\text { net/eng/oportunidades/mercados-inclusivos/ ) }\end{array}$ \\
\hline ENDEVA & $\begin{array}{l}\text { Negocios que incluyen a las personas que viven en condición de pobreza como } \\
\text { productores y consumidores, haciendo una contribución positiva al desarrollo de los } \\
\text { negocios, la población local y el medio ambiente. (ENDEVA, 2009) }\end{array}$ \\
\hline
\end{tabular}

Fuente: Construcción propia basada en fuentes citadas

De esta manera, siendo empresas sostenibles, los NI tienen en consideración los tres pilares de la sostenibilidad, garantizando su factibilidad económica, ambiental y social.

\section{Modos de implementación de un negocio inclusivo}

Como fue propuesto por Lariú y Strandberg (2009), es posible identificar tres formas o modos de implementar un negocio inclusivo, dependiendo del papel jugado por la población de bajos ingresos, a saber:

- 1er modo: Vincula el segmento de la base de la pirámide como un consumidor.

Cuadro No. 2. Aspectos clave de los negocios inclusivos

$\mathrm{Si}$

$\checkmark \quad$ Propone soluciones sostenibles para las necesidades de los pobres.

$\checkmark \quad$ Utiliza las fuerzas del Mercado para el beneficio mutuo.

$\checkmark$ Garantiza la provision de bienes y servicios en mercados desatendidos.

$\checkmark$ Vincula a la población de la BdP como participantes activos de la cadena de valor.

$\checkmark$ Se enfoca en procesos de coinvención y co-creación con la población de la BdP

$\checkmark$ (Simanis \& Hart, 2008).
- 2do modo: Vincula el segmento de la BdP como productores, proveedores o distribuidores.

- 3er modo: Vincula el segmento de la base de la pirámide como un socio o aliado. "Es un proceso de cocreación de nuevos negocios con poblaciones de bajos ingresos".

A manera de síntesis, el Cuadro No. 2 presenta una distinción entre los aspectos principales que corresponden con un negocio inclusivo y aquellos que no.

Fuente: Construcción propia

No

X Se lucra de la pobreza.

× Depende de acciones filantrópicas y donaciones altruistas.

X Corresponde con esquemas de negocios tradicionales.

x Se limita a la interpretación de la BdP como potenciales consumidores.

× Desarrolla actividades dañinas para el medio ambiente. 
Como se desprende del análisis del cuadro anterior, un aspecto crucial para el éxito de los modelos de NI es la creación de espacios de cooperación y desarrollo de esfuerzos conjuntos, beneficiándose de las sinergias que surgen durante el proceso. La sostenibilidad de este tipo de modelos de negocio para la base de la pirámide depende, en gran medida, de la posibilidad de permitir de manera real y efectiva la cocreación de ideas de negocio entre la compañía líder, la comunidad y otros potenciales aliados clave que pueden proveer la asistencia técnica, financiera o de desarrollo humano necesaria para cristalizar el modelo de negocio.

\section{Conclusiones}

Desde la incepción del concepto de Base de la Pirámide hace tan sólo una década, el concepto ha evolucionado de una interpretación puramente comercial de la población en condición de pobreza, hacia una interpretación más holística y multidimensional que percibe a las comunidades como actores tanto en su rol de consumidores como también desempeñando labores de oferta incluyendo la proveeduría o distribución de bienes y servicios. Los modelos de negocios inclusivos, entendidos como aquellos que bajo una lógica de mutuo beneficio permiten vincular a la población pobre en las cadenas de valor de las empresas para beneficios de ambas, se constituyen en una respuesta empresarial viable y sostenible para mejorar las condiciones de vida de la población mundial en situación de pobreza relativa.

De la misma manera, superando la visión puramente asistencialista de la RSE tradicional, los NI logran vincular el desarrollo sostenible con la superación de la pobreza, toda vez que representan esquemas empresariales que permiten la generación de réditos económicos, con justicia social y eficiencia ambiental. Así pues, el potencial de los negocios inclusivos para superar las llamadas trampas de pobreza yace en su viabilidad y escalabilidad comercial. Los modelos de NI, a diferencia del paternalismo corporativo, empoderan a las comunidades al abrir oportunidades de mercado que responden a necesidades básicas insatisfechas, crean autosuficiencia y generan fuentes estables de ingresos. Pero, para ser realmente sostenibles, los NI no sólo requieren ser socialmente benéficos en términos de mejorar la calidad de vida de las comunidades pobres; sino también deben basar dicha mejora en soluciones ecológicamente viables.

En este escenario, el futuro plantea preguntas significativas, tanto para la academia como para la administración, que deben ser objeto de investigación más profunda de tal forma que se refuerce el avance de este naciente modelo en términos de estrategia de negocios. Quizás una de las preguntas principales es, dada la escala y magnitud de las respuestas de mercado necesarias para lograr la inclusión de dos tercios de la humanidad, ¿cómo alcanzar soluciones de negocio sostenibles para todos los ciudadanos del mundo que viven actualmente en la BdP?

En otras palabras, el mayor reto que yace en el futuro es cómo balancear el crecimiento en la producción y el consumo que implica el desarrollo de nuevos modelos de negocio en la BdP, con la limitada capacidad de carga de los ecosistemas del La Tierra. Porque, tal como fue señalado de manera precisa por el reconocido economista Nicholas Stern, "El desarrollo y el cambio climático son los problemas centrales del siglo 21 . Si el mundo falla en 
alguno de ellos, fallará en ambos" (citado en WBSCD, 2010, pág. 6). Así que, ¿̇ómo balancear el deseo de obtener desarrollo para todos con la capacidad ambiental de la Tierra?

Éste es precisamente el mayor reto planteado hoy al desarrollo de modelos de negocios inclusivos. Una mejor calidad de vida para todos los ciudadanos viviendo en condición de pobreza no puede venir a expensas de un deterioro indiscriminado de los recursos naturales, ya que esto tomaría la misma línea de desarrollo insostenible que no debemos emular. Como tampoco pueden la protección ambiental y la lucha contra el cambio climático darse en aislamiento de los problemas sociales que miles de millones de personas en la base de la pirámide tienen que enfrentar.

Como es evidente, el análisis aquí presentado no pretende ser exhaustivo ni bridar un modelo único para la interpretación de la sostenibilidad en los negocios inclusivos dirigidos a los mercados de la base de la pirámide, reconociendo sus limitaciones al tratarse de un estudio exploratorio de carácter cualitativo. Por el contrario, busca hacer una contribución preliminar a la escasa literatura existente en la materia, sirviendo de base e incentivo para el desarrollo de futuras investigaciones de la misma línea, que permitan probar y complementar las conclusiones y observaciones aquí contenidas. En particular, se recomienda que dichas investigaciones enfaticen en explorar las contradicciones existentes entre el aumento de la producción y el consumo en la $\mathrm{BdP}$, y la protección ambiental.

\section{Bibliografía}

Anderson, J. \& Billou, N. (2007). Serving the World's Poor: Innovation at the Base of the Economic Pyramid, Journal of Business Strategy, 28 (2), 14-21.

Anderson, J. \& Markides, C. (2007). Strategic Innovation at the base of the pyramid, MIT Sloan Management Review, Fall, 82-88.

Barkemeyer, R. (2011). Corporate perceptions of sustainability challenges in developed and developing countries: constituting a CSR divide? Social Responsibility Journal, 7 (2), 257-281.

Berger, R., Choi, C., \& Kim, J. (2011). Responsible Leadership for Multinational Enterprises in Bottom of Pyramid Countries: The Knowledge of Local Managers. Journal of Business Ethics, 101, 553-561

Boada, A., Rocchi, S., Kuhndt, M. (2005). Negocios y Sostenibilidad más allá de la gestión ambiental. Editorial Politécnico Grancolombiano: Bogotá. 155 pp.

Brown, L. R., (2011). El mundo al borde del abismo: ¿Cómo evitar el declive ecológico y el colapso de la economía? Traducción por: Rincón, G., Pineda, M.A. y Másmela, T. Bogotá: Ecoe ediciones.

Canel, E., Idemudia, U., \& North, L. L. (2010). Rethinking extractive industry: Regulation, dispossession, and emerging claims. Canadian Journal of Development Studies, 30(1-2), 5-25.

CECODES-SNV, (2008). Los Negocios Inclusivos en Colombia. Bogotá: CECODES.

CECODES (2010). Cambiando el rumbo. Casos de sostenibilidad en Colombia. Bogotá: CECODES.

CECODES (2011). Sostenibilidad en Colombia. Casos empresariales. Bogotá: CECODES 
Cooke, D. (2010). Building social capital through corporate social investment, AsiaPacific Journal of Business Administration, 2 (1), 71-87.

Dahlsrud, A. (2006). How corporate social responsibility is defined: An analysis of 37 definitions, Corporate Social Responsibility and Environmental Management, 15, 1-13.

Dalby, S. (2013) The geopolitics of climate change, Political Geography, Volume 37, November 2013, pp 38-47.

Eabrasu, M. (2012). A Moral Pluralist Perspective on Corporate Social Responsibility: From Good to Controversial Practices. J Bus Ethics. published online DOI 10.1007/s10551-012-1491-3.

ENDEVA (2009). Inclusive Business Guide, How to Develop Business and Fight Poverty. Berlin: ENDEVA.

Freeman, I. \& Hasnaoui, A. (2011). The Meaning of Corporate Social Responsibility: The Vision of Four Nations, Journal of Business Ethics, 100, 419-443.

Friedman, M. (13 de septiembre de 1970). The social responsibility of business is to increase its profits. New York Times Magazine, pp. 122-126.

Frynas, J.G. (2005). The false developmental promise of corporate social responsibility: evidence from multinational oil companies, International Affairs, 81 (3), 581-98.

Frynas, J.G. (2008). Corporate social responsibility and international development: critical assessment, Corporate Governance, 16 (4), 274-81.

Garriga, E. \& Melé, D. (2004). Corporate Social Responsibility Theories: Mapping the Territory, Journal of Business Ethics, 53, 51-71.

Gates, D. \& Steane, P. (2009). Altruism-an alternative value in policy formation and decision making, International Journal of Social Economics, 36 (10), 962-978.
Goodland, R. (1994). El argumento según el cual el mundo ha llegado a sus limites en Goodland, Robert et al. (2003). Desarrollo Económico Sostenible. Bogotá: Tercer Mundo Editores.

Gond, J-P. \& Moon, J. (2011). Corporate Social Responsibility in Retrospect and Prospect: Exploring the Life-Cycle of an Essentially Contested Concept, in J-P Gond and J Moon eds Corporate Social Responsibility: A Reader Routledge. Available as ICCSR Research Paper No. 59, 1-40.

Hahn, R. (2009). The Ethical Rationale of Business for the Poor-Integrating the Concepts Bottom of the Pyramid, Sustainable Development, and Corporate Citizenship, Journal of Business Ethics, 84, 313-324.

Hahn, R. (2012). Inclusive business, human rights and the dignity of the poor: a glance beyond economic impacts of adapted business models, Business Ethics: A European Review, 21 (1), 47-63

Halme, M., Linderman, S., \& Linna, P. (2012). Innovation for Inclusive Business: Intrapreneurial Bricolage in Multinational Corporations. Journal of Management Studies, 1- 42.

Hammond, A. e. (2007). The Next 4 Billion: Market Size and Business Strategy at the Base of the Pyramid. Washington D.C.: World Resources Institute and International Finance Corporation.

Hart, S. (2010). Capitalism at the Crossroads. Next generation business strategies for a post-crisis world ( $3^{\text {rd }}$ ed.). New Jersey: Wharton School Publishing

Hart, S. and Christensen, C. (2002). The Great Leap, driving innovation from the base of the pyramid. MIT Sloan Management Review, Fall, 51-56.

Hart, S. and London, T. (2005). Developing native capability: What multinational corporations can learn from the base of the 
pyramid. Standford Social Innovation Review, Summer, 28-33.

Hawken, P., Lovins, A., Lovins, H. (1999). Natural Capitalism: Creating the Next Industrial Revolution. Little Brown and Company 396 pp.

Henderson, D. (2001). The case against 'corporate social responsibility'. Policy, 17(2), 28-32.

HiRSEh, P. M. \& Levin, D. Z. (1999). "Umbrella Constructs Versus Validity Police: A Life Cycle Model”, Organization Science, 10 (2), 199-212.

ISO (2010). ISO 26000:2010 Guidance on social responsibility, 106. Geneve, Switzerland: ISO.

Ite, U. E. (2005). Poverty reduction in resource-rich developing countries: what have multinational corporations got to do with it? Journal of International Development, 17 (7), 913-929.

Jamali, D. (2010). The CSR of MNC Subsidiaries in Developing Countries: Global, Local, Substantive or Diluted?, Journal of Business Ethics, 93,181-200.

Jenkins, B. \& Eriko, I. (2009). Business Linkages: Enabling Access to Markets at the Base of the Pyramid. Report of a Roundtable Dialogue March 3-5, 2009, Jaipur, India. Washington, DC: International Finance Corporation, International Business Leaders Forum, and the CSR Initiative at the Harvard Kennedy School.

Jenkins, B., Ishikawa, E., Geaneotes, A., \& Paul, J. (2010). Inclusive Business: Expanding Opportunity and Access at the Base of the Pyramid. Report of a Conference held October 7-8, 2010 in Washington, DC. Washington, DC: IFC.

Jeppesen, S. \& Lund-thomsen, P. (2010), Special Issue on "New Perspectives on Business, Development, and Society Research, Journal of Business Ethics, 93, 139-142.
Kandachar, P. \& Halme, M. (2008). Sustainability Challenges and Solutions at the Base of the Pyramid. Sheffield: Greenleaf Publishing.

Karnani, A. (2007). Misfortune at the Bottom of the Pyramid, Greener Management International, 51, 99-110.

Kolk, A. \& van Tulder, R. (2006). Poverty Alleviation as Business Strategy? Evaluating Commitments of Frontrunner Multinational Corporations, World Development, 34 (5), 789-801.

Lariú, A. \& Strandberg, L. (2009). Negocios Inclusivos: Creando valor para las empresas y para la población de bajos ingresos. Cátedra "la Caixa" de Responsabilidad Social de la Empresa y Gobierno Corporativo.

Leonard, A. (2010). La Historia De Las Cosas. Fondo de cultura económica de España, S.L. 392 pp.

Ludevid, M. (1997) El cambio global en el medio ambiente: introducción a sus causas humanas. Barcelona. Marcombo Boixareu Editores.

Lodge, G. C., \& Wilson, C. (2006). A Corporate Solution to Global Poverty: How Multinationals Can Help the Poor and Invigorate Their Own Legitimacy. Princeton University Press.

Maak, T. (2009). The cosmopolitan corporation. Journal of Business Ethics, 84, 361-372.

Mendoza, R. y Thelen, N. (2008). Innovations to make markets more inclusive for the poor. Development Policy Review, 26 (4), 427-458.

Montes, A. (2013). Análisis de la contribución de los sanitarios secos al saneamiento básico rural. Caso: vereda chorillos. Punto de Vista, IV, 7, 133 -148. 
Muthuri, J. N., Moon, J., \& Idemudia, U. (2012). Corporate Innovation and Sustainable Community Development in Developing Countries. Business Society, 51 (3), 355-381.

Prahalad, C. K., \& Hart, S. (2002). The Fortune at the bottom of the pyramid. Strategy+ Business, 26, 54-67.

Prahalad, C.K., \& Hammond, A. (2002). Serving the world's poor profitably. Harvard Business Review, 4-11.

Prahalad, C.K. (2005). The Fortune at the Bottom of the Pyramid: Eradicating Poverty through Profits. New Jersey: Wharton School Publishing.

Programa de las Naciones Unidas para el Desarrollo, PNUD (2010). Crecimiento de mercados inclusivos, Bogota: PNUD.

Reich, R. B. (1998). The New Meaning of Corporate Social Responsibility. California Management Review, 40 (2), 1-11.

Schuster, T., \& Holtbrügge, D. (2012). Market entry of multinational companies in markets at the bottom of the pyramid: $A$ learning perspective. International Business Review, 21, 817-830.

Sen, A. \& Kliksberg, B. (2007). Primero la Gente. Barcelona: Ediciones Deusto.

Simanis, E. and Hart, S. (2008). The Base of the Pyramid Protocol: Toward Next Generation BoP Strategy.(2nd ed.). New York: Cornell University.

Simanis, E. and Hart, S. (2009). Innovation from the inside out. Sustainability \& Innovation MIT Sloan Management Review, Summer, 77-86.

Singer, A. (2006). Business strategy and poverty alleviation. Journal of Business Ethics, 66, 225-231.
SNV y WBCSD (2008). Negocios Inclusivos Iniciativas empresariales rentables con impacto en el desarrollo. Quito: WBCSD Stigson, B., \& Rendlen, B. (2006). Drivers of Business Behaviour in the Realm of Sustainable Development: The Role and Influence of the WBCSD, a Global Business Network. In F. Wijen, K. Zoeteman, $\&$ J. Pieters, A Handbook of Globalisation and Environmental Policy: National Government Interventions in a Global Arena (pp. 313-332). Cheltenham: Edward Elgar Publishing.

Tashman, P. \& Marano, V. (2010). Dynamic Capabilities and Base of the Pyramid Business Strategies. Journal of Business Ethics, 89, 495-514.

VanSandt, C.V., \& Sud, M. (2012). Poverty Alleviation through Partnerships: A Road Less Travelled for Business, Governments, and Entrepreneurs. Journal of Business Ethics,110, 321-332.

Velásquez, F. (2008) ¿Es posible la sostenibilidad? Reflexiones sobre el medio ambiente. Madrid. Editorial popular.

Visser, W. Matten, D. Pohl, M. \& Tolhurst, N. 2010, The $A-Z$ of corporate social responsibility. Rev. and updated ed. Great Britain: Wiley.

Whelan, G., Moon, J. \& Orlitzky, M. (2009). Human Rights, Transnational Corporations and Embedded Liberalism: What Chance Consensus? Journal of Business Ethics, 87, 367-383.

World Business Council for Sustainable Development, WBCSD. 2010, Business and development. Challenges and opportunities in a rapidly changing world. Geneva: WBCSD. 